\title{
A dynamic preferred direction model for the self-organization dynamics of bacterial microfluidic pumping
}

\author{
Daniel Svenšek ${ }^{1}$, Harald Pleiner ${ }^{2}$, and Helmut R. Brand ${ }^{3}$ \\ 1 Department of Physics, Faculty of Mathematics and Physics, \\ University of Ljubljana, SI-1000 Ljubljana, Slovenia \\ 2 Max Planck Institute for Polymer Research, POBox 3148, 55021 Mainz, Germany \\ 3 Theoretische Physik III, Universität Bayreuth, 95440 Bayreuth, Germany
}

(Dated: January 4, 2019)

\begin{abstract}
It is known that some flagellated bacteria like Serratia marcescens, when deposited and affixed onto a surface to form a "bacterial carpet", self-organize in a collective motion of the flagella that is capable of pumping fluid through microfluidic channels. We set up a continuum model comprising two macroscopic variables, that is capable of describing this self-organization mechanism as well as quantifying it to the extent that an agreement with the experimentally observed channel width dependence of the pumping is reached. The activity is introduced through a collective angular velocity of the helical flagella rotation, which is an example of a dynamic macroscopic preferred direction. Our model supports and quantifies the view that the self-coordination is due to a positive feedback loop between the bacterial flagella and the local flow generated by their rotation. Moreover, our results indicate that this biological active system is operating close to the self-organization threshold.
\end{abstract}

\section{INTRODUCTION}

Since the pioneering work by Kim and Breuer on self-organizing bacteria carpets leading to microfluidic pumping [1-5], the phenomena of bacterial self-organization resulting in spontaneous fluid flow are meanwhile readily reproduced and are already exploited on a level approaching applications [6-12]. In a qualitative picture, the mechanism responsible for all these spontaneously driven flows is considered to be collective coordination between individual bacteria caused by fluid flow that is generated by their flagella. This is a class of biological actively propelled systems, where examples are ranging from molecular motors-microtubules/filaments mixtures [13-20], bacteria [21-25], sperm cells [26, 27], to insects [28, 29], fish [30, 31], birds [32-35], and herds [36].

In this contribution, we focus on the fundamental question of the mechanism and properties of the bacterial selforganization producing fluid flow. We take as specific input the original experiment on the microfluidic pump powered by self-organizing bacteria described in detail in Ref. [5]. Besides its groundbreaking importance, another particular merit of this work lies in the fact that it provides enough information that a quantitative correspondence with a generic continuum model involving several parameters can be established.

Activity is usually accompanied by some kind of propulsion, therefore it is naturally connected with a preferred direction the system might already possess or acquire due to activity. There exist diverse ways of introducing activity into an otherwise passive yet orientationally ordered system. Frequently this is done tout de suite via an "active term" in the stress tensor [37], generating extensile ("pullers") or contractile ("pushers") stress proportional to the static nematic preferred direction. A nonzero divergence of this stress tensor generates fluid flow described within the framework of nematodynamics, i.e., hydrodynamics of nematic liquid crystals. It has been shown that such flow-driving contribution to the stress tensor indeed arises - accompanied by counter terms - from hydrodynamic theories incorporating a static preferred direction coupled to a chemical reaction [38], or introducing a so-called dynamic preferred direction $[39,40]$ which is a time rate, i.e., it changes sign if time is reversed.

The concept of the dynamic preferred direction $[41,42]$ is naturally suited for systems exhibiting orientational order dynamically rather than statically. Flocking biological systems like bird flocks, fish schools, insect swarms, moving bacterial colonies are good examples. In all of these, the preferred direction is the collective velocity of active units. It is evident that in these systems the orientational order can exist only dynamically and disappears as soon as the motion ceases (e.g., birds resting in the trees or on the ground, bees forming a cluster, colony of immotile bacteria). This is to be contrasted with the static, "ever-persisting" orientational order of necessarily elongated or flattened elements, e.g., in thermo- or lyotropic nematic liquid crystals. A further example of a dynamic preferred direction with a different inversion symmetry is the collective angular velocity, e.g., of flagella of (usually) prokaryotic cells (bacteria). Angular velocity is symmetric with respect to inversion of space, as opposed to the velocity from previous examples which is antisymmetric.

Certainly, the precondition for a sensible continuum description is a sufficiently large number of locally ordered active units such that coarse graining is possible. In the last years, however, awareness is growing that for animals like birds, fish or insects this is probably not the severest hinderance to the continuum description. The substantial problem lies in the fact that these are advanced organisms with a nervous system and thus with complex sensing, 
processing and reacting abilities, whose intelligent reactions are beyond simple response to changes that take place locally in space and time (cf. Ref. [35] for bird flocks). The latter is however just the basic assumption of the continuum approach using partial differential equations that are local in space and time by definition.

Collective dynamics of bacteria, on the other hand, seems to be considerably better suited for continuum modeling (cf. e.g. Ref. [24]). Bacteria are large in numbers, but most importantly they are primitive and react to local changes only, with a reaction that is direct, simple and dully reproducible.

\section{BRIEF SUMMARY OF KEY EXPERIMENT}

In the bacterial pumping experiment of Ref. [5], they used fully enclosed polydimethylsiloxane (PDMS) microfluidic channels of length (a) $15 \mathrm{~mm}$, height $c=15 \mu \mathrm{m}$ and width $b$ ranging from 50 to $800 \mu \mathrm{m}$. Bacterial carpets were formed on the inner surfaces of the channel using a flow-deposition procedure in which the prolate bodies of the bacteria adhered to the surfaces by natural accretion with a flow-induced orientational preference along the channel axis and several flagella free to rotate in the fluid. At its ends, the channel had an inlet and outlet at strictly zero pressure difference. The velocity of the fluid was measured at a fixed point at the midpoint of the channel using particle tracking velocimetry. The tracer particles were always observed to move in the same direction and this "left-right" bias was attributed to the carpet fabrication sequence.

The key result is that the system of thousands of independent bacterial cells self-organizes, resulting in a collective motion that is capable of pumping fluid through the microchannel. Noise is recognized as an important feature and is also quantified experimentally in considerable detail. The measured root-mean-square (rms) fluctuations of the flow velocity amount to $30-50 \%$ of the average flow velocity. Moreover, streamwise velocity fluctuations are systematically larger than spanwise fluctuations.

As already pictured by the authors, a positive feedback mechanism between the flagella and the local flow generated by their rotation is considered crucial for the growth of coordinated regions. Besides the very existence of the selforganization, the main experimental result is its well-defined dependence on the width of the microchannel: the velocity of pumping consistently decreases with increasing channel width.

\section{MODEL}

We present a lean continuum model comprising two macroscopic variables, that is capable of describing this selforganization feedback mechanism as well as the observed dependence of the pumping on the channel width. We consider a thin channel with $x$ the coordinate along its length $(-a / 2<x<a / 2), y$ the coordinate across its width $(-b / 2<y<b / 2)$, and height $c \ll b<a$, Fig. 1. One of the variables, which is also monitored in experiment, is the two-dimensional (2D) macroscopic in-plane velocity of the pumped fluid, $\mathbf{v}=v_{x}(x, y) \hat{\mathbf{e}}_{x}+v_{y}(x, y) \hat{\mathbf{e}}_{y}$, satisfying the incompressibility condition $\nabla \cdot \mathbf{v}=0$ at constant density $\rho$ of the fluid. The activity is introduced by another variable, which is a $2 \mathrm{D}$ collective (average, coarse-grained) angular velocity of the helical flagella rotation, $\mathbf{W}=$ $W_{x}(x, y) \hat{\mathbf{e}}_{x}+W_{y}(x, y) \hat{\mathbf{e}}_{y}$. The speed of rotation of an individual flagellum (or a small group belonging to an individual bacterium) is considered constant. What varies, however, is the collective angular velocity: $\mathbf{W}=0$ means that the individual flagella are pointing in random directions, whereas $\mathbf{W} \neq 0$ corresponds to orientational order of the flagella, i.e., collective rotation. The quantity $\mathbf{W}$ is an example of a dynamic preferred direction, which is a time rate and thus changes sign when time is reversed, as opposed to a static preferred direction (e.g. the director of a nematic liquid crystal) that is even under time reversal. Like any angular velocity, $\mathbf{W}$ is an axial vector (it does not change sign upon inversion of space).

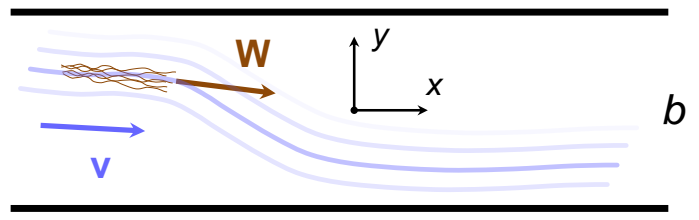

a

FIG. 1. Schematic top view of the microchannel with two-dimensional fluid velocity field $\mathbf{v}$ and active orientational field $\mathbf{W}$ of the bacterial flagella. The thickness of the microchannel is small compared to its length $a$ and width $b$. 
A minimal model coupling the variables $\mathbf{v}(x, y, t)$ and $\mathbf{W}(x, y, t)$ is

$$
\begin{aligned}
\frac{\partial \mathbf{v}}{\partial t} & =-\frac{1}{\rho} \nabla p+D_{v} \nabla^{2} \mathbf{v}-\frac{1}{\tau_{v}} \mathbf{v}-\gamma_{v} v^{2} \mathbf{v}+\alpha_{v} \mathbf{W}, \\
\frac{\partial \mathbf{W}}{\partial t}+\frac{1}{2} \mathbf{W} \times(\nabla \times \mathbf{v}) & =D_{W} \nabla^{2} \mathbf{W}-\frac{1}{\tau_{W}} \mathbf{W}-\gamma_{W} W^{2} \mathbf{W}+\alpha_{W} \mathbf{v}+\boldsymbol{\zeta}(x, y, t),
\end{aligned}
$$

where $v^{2}=|\mathbf{v}|^{2}, W^{2}=|\mathbf{W}|^{2}, p(x, y, t)$ is the pressure and $\boldsymbol{\zeta}(x, y, t)$ is stochastic noise with average power $\left\langle\left|\boldsymbol{\zeta}^{2}\right|\right\rangle \equiv 2 q$.

We now explain in due order the physics contained in the model Eqs. (1)-(2). Without the coupling, the fields are relaxing to zero with characteristic times $\tau_{v}$ and $\tau_{W}$. The relaxation of the velocity field is due to the viscous dissipation coming from the velocity gradient across the thin channel. The relaxation of $\mathbf{W}$ describes the absence of spontaneous orientational ordering of the flagella without the ordering effect of the velocity field. The cubic $\gamma_{v}, \gamma_{W}$ terms are required to limit the amplitudes of both variables to finite values, which certainly takes place in reality. (We do not aim to discuss the various possible saturation mechanisms giving rise to these and general higher order terms, pointing out an obvious one as an example: there is an upper limit to $W$, reached when all flagella are aligned.) The $D_{v}$ term describes the in-plane viscous dissipation, while the $D_{W}$ term is a generic Laplacian analogous to the director elastic term in nematic liquid crystals [43], taking into account local interaction between the flagella. The advective derivative $(\mathbf{v} \cdot \nabla) \mathbf{v}$ is omitted from Eq. (1) since the empirical Reynolds number is of the order of $10^{-4}$, and from Eq. (2) since the bacteria, once deposited, are fixed to the substrate and thus $\mathbf{W}$ is not advected. The corotation derivative $\frac{1}{2} \mathbf{W} \times(\nabla \times \mathbf{v})$, however, cannot be discarded in principle. In the absence of other forces, $\mathbf{W}$ would locally rotate with the flow, which is described by this term. Its influence will be discussed in Sec. IV B. The cross coupling between the velocity $\mathbf{v}$ and the angular velocity $\mathbf{W}$ is possible, since the flagella are helical ("propellers") and thus break inversion symmetry, i.e., the coupling parameters $\alpha_{v}$ and $\alpha_{W}$ are pseudoscalars, changing sign upon inversion. With that, Eqs. (1)-(2) respect inversion symmetry. Noise is applied to the $\mathbf{W}$ variable and is considered primarily of active, biological origin. It captures both directional fluctuations and the known sporadic reversals of the sense of rotation of individual flagella [5].

The actual scale of the $\mathbf{W}$ variable is unimportant as it will not be directly connected to the physical angular velocity or, rather, angular momentum density of the flagella. Moreover, Eq. (1) can be rescaled by the physical scale of the velocity. The only spot where the physical velocity scale actually matters is the corotational derivative of Eq. (2), which we will handle specifically. Hence, we will consider both variables $\mathbf{W}$ and $\mathbf{v}$ dimensionless.

\section{A. Homogeneous solutions}

First we characterize the basic properties and solutions of the model Eqs. (1)-(2). For a sufficiently strong cross coupling,

$$
\alpha_{v} \alpha_{W}>\frac{1}{\tau_{v}^{k} \tau_{W}^{k}}
$$

where $1 / \tau_{\{v, W\}}^{k}=1 / \tau_{\{v, W\}}+D_{\{v, W\}} k^{2}$, the state $\mathbf{v}=0, \mathbf{W}=0$ becomes linearly unstable against a perturbation with $\mathbf{v}$ and $\mathbf{W}$ parallel and perpendicular to the wavevector $\mathbf{k}$ of the perturbation, and grows with the rate

$$
\frac{1}{\tau_{k}}=\frac{1}{2}\left[-\left(\frac{1}{\tau_{W}^{k}}+\frac{1}{\tau_{v}^{k}}\right)+\sqrt{\left(\frac{1}{\tau_{W}^{k}}-\frac{1}{\tau_{v}^{k}}\right)^{2}+4 \alpha_{v} \alpha_{W}}\right] .
$$

The threshold Eq. (3) is lowest for a homogeneous perturbation $(\mathbf{k}=0)$, for which also the growth rate Eq. (4) is highest. This long-wavelength $(\mathbf{k} \rightarrow 0)$ instability implies that the model indeed incorporates a positive feedback mechanism between $\mathbf{v}$ and $\mathbf{W}$ leading to macroscopic coordination for $\alpha_{W} \tau_{W} \alpha_{v} \tau_{v}>1$. Notwithstanding the fact that the collective angular velocity $\mathbf{W}$ of the flagella is not measured experimentally, the existence of the self-coordination threshold as a result of its coupling with $\mathbf{v}$ speaks for the physical relevance of this variable.

Far above the threshold Eq. (3), i.e., in the limit of strong coupling, such that $\gamma_{W} W_{0}^{2} \gg 1 / \tau_{W}, \gamma_{v} v_{0}^{2} \gg 1 / \tau_{v}$, the amplitudes of the homogeneous stationary state are

$$
\begin{aligned}
W_{0}^{2} & =\left(\frac{\alpha_{W}^{3} \alpha_{v}}{\gamma_{W}^{3} \gamma_{v}}\right)^{1 / 4} \\
v_{0}^{2} & =\left(\frac{\alpha_{v}^{3} \alpha_{W}}{\gamma_{v}^{3} \gamma_{W}}\right)^{1 / 4} .
\end{aligned}
$$


Conversely, only slightly above the threshold, i.e., for weakly supercritical coupling, such that

$$
\gamma_{W} W_{0}^{2} \ll 1 / \tau_{W} \quad \gamma_{v} v_{0}^{2} \ll 1 / \tau_{v},
$$

the amplitudes of the homogeneous stationary state are

$$
\begin{aligned}
W_{0}^{2} & =\left(\tau_{W} \gamma_{W}+\frac{\tau_{v} \gamma_{v}}{\tau_{W}^{2} \alpha_{W}^{2}}\right)^{-1}\left(\tau_{W} \alpha_{W} \tau_{v} \alpha_{v}-1\right), \\
v_{0}^{2} & =\left(\tau_{v} \gamma_{v}+\frac{\tau_{W} \gamma_{W}}{\tau_{v}^{2} \alpha_{v}^{2}}\right)^{-1}\left(\tau_{W} \alpha_{W} \tau_{v} \alpha_{v}-1\right),
\end{aligned}
$$

i.e., they grow continuously from the onset.

\section{B. Connection with the experiment}

The model, Eqs. (1) and (2), is rather comprehensive and can furnish several theoretical regimes of dynamics, where not all of them can be relevant for the realistic situation. Taking into account experimental facts, one can however narrow down the parameter space accordingly and arrive at a regime that closely mimics the real system. Although this part may appear technical, it is profoundly physical and is vital for a description that is relevant.

Due to the small thickness $c$ of the microchannel, the velocity field relaxes on a characteristic time scale as short as

$$
\tau_{v} \sim \frac{\rho c^{2}}{\pi^{2} \eta} \approx 20 \mu \mathrm{s}
$$

where $\rho \approx 1000 \mathrm{~kg} / \mathrm{m}^{3}$ is the density and $\eta \approx 0.001$ Pas the viscosity of the aqueous solution. For the same reason the relative magnitude of the in-plane viscous term is small,

$$
D_{v} \nabla^{2} v \times\left(\frac{1}{\tau_{v}} v\right)^{-1} \sim \frac{c^{2}}{b^{2}} \ll 1
$$

where $D_{v}=\eta / \rho$ and $b$ is the width of the microchannel.

On the other hand, the characteristic time of the $\mathbf{W}$ variable, $\tau_{W}$, is much larger. Based on the available data it should be in the range from $10^{-1} \mathrm{~s}$ to several $10^{2} \mathrm{~s}$. Hence, we arrive at an important point, which is not obvious judging from the similar form of Eqs. (1)-(2) - the model is clearly asymmetric with respect to the time scales: W is the slow variable defining the overall time scale of the dynamics, while $\mathbf{v}$ adapts almost instantaneously and can therefore be treated as quasi-stationary, $\partial \mathbf{v} / \partial t=0$. Consequently, $\tau_{v}$ is a redundant parameter and is absorbed in the remaining parameters of Eq. (1). For the reason of trackability we will however keep it and put it to $\tau_{v}=1$ from now on. Similarly, $\tau_{W}$ will be used as the time unit in Eq. (2). In these units, $\tau_{W}=1$ holds. For trackability we again retain the symbol $\tau_{W}$ everywhere.

In the limit of the quasi-stationary velocity field, the self-organization growth rate $1 / \tau_{k}$, Eq. (4), here for $k=0$, is

$$
\frac{1}{\tau_{0}}=\frac{1}{\tau_{W}}\left(\alpha_{W} \tau_{W} \alpha_{v} \tau_{v}-1\right) .
$$

We see that the driving is controlled by the product $\alpha_{W} \tau_{W} \alpha_{v} \tau_{v}$, where

$$
\alpha_{W} \tau_{W} \alpha_{v} \tau_{v}=1
$$

corresponds to the threshold for the self-organization. In the absence of any other empirical input we consider symmetric driving $\alpha_{W} \tau_{W}=\alpha_{v} \tau_{v}$.

Since the scale of the dimensionless variables $\mathbf{W}$ and $\mathbf{v}$ has no physical meaning, we will cap them to values close to unity by demanding in Eqs. (5) and (6) $W_{0}=v_{0}=1$. This fixes

$$
\gamma_{W}=\left|\alpha_{W}\right|, \quad \gamma_{v}=\left|\alpha_{v}\right| .
$$

The actual magnitude of the corotation term of Eq. (2) will be taken into account by scaling this term with the physical velocity scale $v_{s}$, which thus becomes an empirical parameter. This is the only spot where the actual velocity scale is relevant. We use $v_{s} \sim 50 \mu \mathrm{m} / \mathrm{s}$, which is the maximum speed at which an individual bacterium of 
the considered type is propelled through water [5]. The estimate of the magnitude of the corotation term is then $|\mathbf{W} \times(\nabla \times \mathbf{v})| v_{s} \sim v_{s} \tau_{W} / b$ and ultimately depends on the physical scale of the characteristic time $\tau_{W}$.

The two remaining parameters - the diffusivity $D_{W}$ of the $\mathbf{W}$ field and the noise strength $q$ - are particularly important. Their role is explored more closely in Appendix A, defining two characteristic length scales. In bulk and in the absence of noise, the "correlation length" $\xi \sim \sqrt{\tau_{0} D_{W} / 2}$ would be the characteristic size of vortices in the $\mathbf{v}$ and $\mathbf{W}$ fields. It depends on the ratio of the elasticity (diffusivity) $D_{W}$ and the linear growth rate $1 / \tau_{0}$, describing the distance from the threshold, Eq. (12). The other characteristic length, related to noise - a "persistence length" $\lambda_{Q} \sim \sqrt{2 \pi^{2} D_{W} / Q}$ of the self-organized order — can be interpreted as the length over which the boundary-induced ordering of the flagella persists before it is destroyed by noise with strength $Q$, Eq. (B5). In the most relevant regime, where the flow morphology is governed by the competition between diffusion (elasticity) of the $\mathbf{W}$ field and noise acting on it, $\lambda_{Q}$ represents the key length scale of the system and is to be compared to the width of the microchannel.

\section{Physical essence of the model}

It turns out that the left-right asymmetry, i.e., the absence of global flow direction reversals, cannot be realistically reproduced by introducing a simple bulk bias to either of the equations Eqs. (1) or (2). Namely, a constant bias force along $\hat{\mathbf{e}}_{x}$, exerted on $\mathbf{W}$ or $\mathbf{v}$, suppresses the effect of noise for wider channels and enhances pumping, which is contrary to the main experimental result. This point is crucial. Reducing the bias to avoid this unwanted effect, flow reversals take place on a regular basis, which is also not reported. If one insisted on a bulk bias, a strong nonlinear dependence of the $\mathbf{W}$ bias force on the angle between $\mathbf{W}$ and $\hat{\mathbf{e}}_{x}$ would presumably improve the situation. This is however delicate and would require specific experimental input. Instead, we fix $\mathbf{W}$ at the channel walls $y= \pm b / 2$ in the $\hat{\mathbf{e}}_{x}$ direction and with a nonzero magnitude, thus bringing in the left-right bias as a boundary effect. Moreover, bulk self-organization is predominantly enforced by the boundaries in this picture, while the pumping efficiency depends primarily on the parameters $D_{W}$ and $Q$ through the ratio of $\lambda_{Q}$ to the width $b$ of the channel. One should bear in mind that nevertheless being by far the simplest, this is not the only explanation that is eventually possible.

Extrapolating further this simplified picture, the self-organization mechanism is explained as a boundary-induced ordering phenomenon ("wetting"), where there is a competition between the elasticity (or diffusivity) of the system, $D_{W}$, promoting the ordering effect of the boundaries, and the bulk disordered phase $\mathbf{W}=\mathbf{v}=0$. Note that the system is disordered in bulk, as displayed by the vanishing pumping in channels of increasing width. In this simplest mean-field picture, noise does not appear explicitly but is however responsible for the disordered ground state.

In our description, we include noise and model the fluctuations explicitly, since the velocity fluctuations are well characterized experimentally. In particular, the empirical distinction between the magnitude of the streamwise and spanwise velocity fluctuations is a valuable item of information as we will demonstrate. In the absence of noise, the bulk phase is ordered in our model, and becomes disordered due to the action of noise. The distance from the self-organization threshold Eq. (13) is a delicate parameter, and the ambition is to connect it with the physical system if possible.

\section{Method and boundary conditions}

Dynamic equations Eqs. (1) and (2) are advanced in time by the Euler method on a 2D mesh discretizing the channel in $x$ and $y$ directions. One might be tempted to use a coarser meshing along the length of the microchannel, which however interferes with the circular vortex structure of both fields and impairs the solutions significantly. Therefore, the mesh is kept strictly equilateral in all cases to avoid such anisotropic artifacts. The incompressibility condition is satisfied by an artificial compressibility approach, i.e., using a dynamic equation for the density of the fluid with the time scale that is short with respect to all physical time scales. Details of the method and noise application are presented in Appendix B.

At the long sides of the microchannel $(y= \pm b / 2), v_{x}=v_{y}=0$ is required. At the short sides $(x= \pm a / 2)$, zero gradient boundary condition $\partial \mathbf{v} / \partial x=0$ is applied. The boundary condition for the density is switched between inlet and outlet depending on the sign of $v_{x}$ : at the left boundary we require $\rho(-a / 2, y)=1$, if $v_{x} \geq 0$, and $\partial \rho / \partial x(-a / 2, y)=0$ otherwise, and analogously at the right boundary. It is important that no left-right bias is introduced by the density boundary conditions.

As explained in Sec. III C, at $y= \pm b / 2$ we fix $\mathbf{W}=W_{0} \hat{\mathbf{e}}_{x}$, where $W_{0}$ is the actual bulk equilibrium value of $|\mathbf{W}|$ in the absence of noise (Eqs. (5) and (8) are strong- and weak-coupling limits of the magnitude used here). At $x= \pm a / 2$, zero gradient boundary condition $\partial \mathbf{W} / \partial x=0$ is applied. 

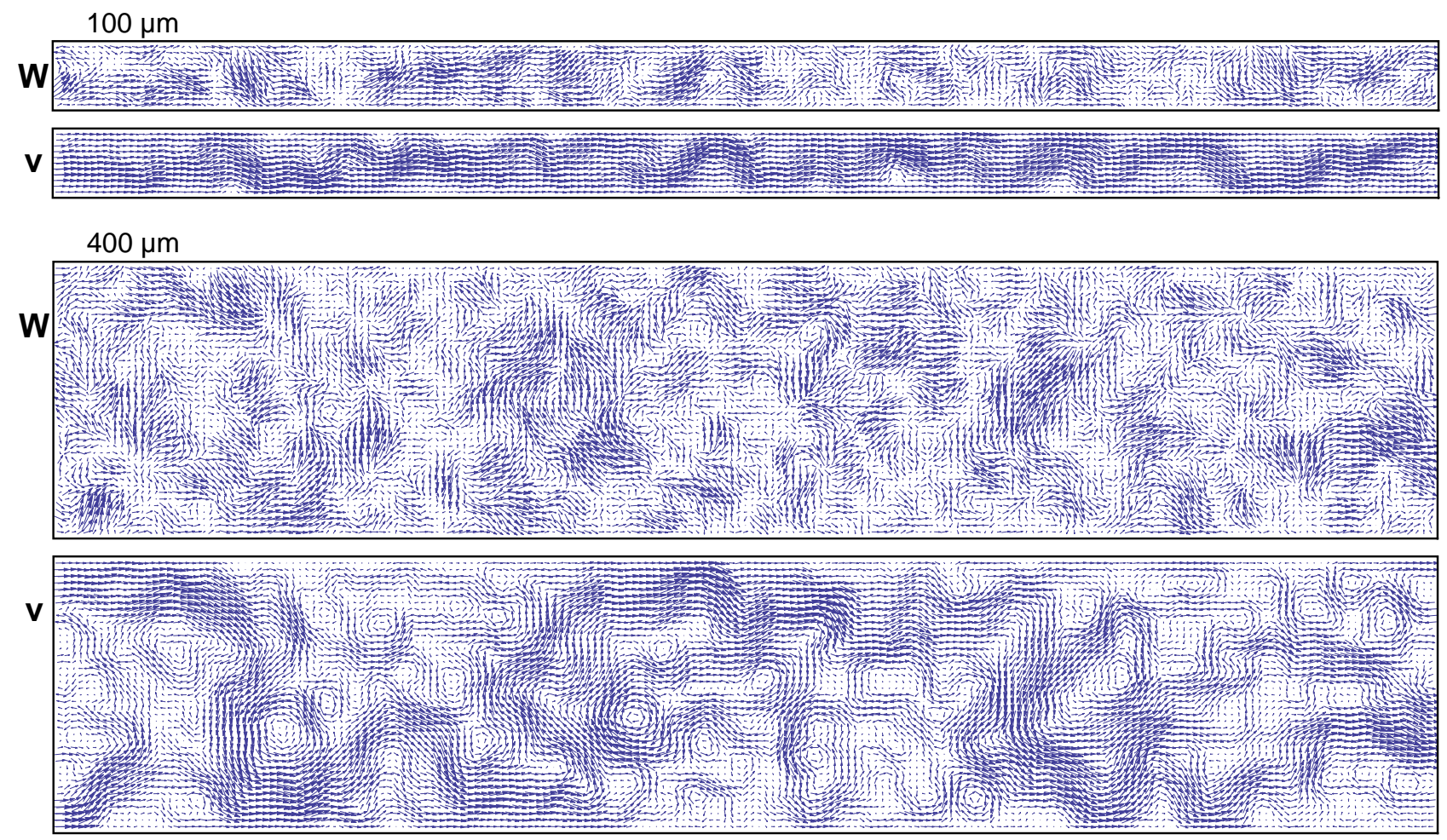

FIG. 2. Snapshots of flagella angular velocity, $\mathbf{W}(x, y)$, and corresponding fluid velocity, $\mathbf{v}(x, y)$, fields in $2 \mathrm{~mm}$ long microchannels of width $b=100 \mu \mathrm{m}$ (top) and $b=400 \mu \mathrm{m}$ (bottom). The stream-like nature of the velocity fields $(\nabla \cdot \mathbf{v}=0)$ contrasts well with the W fields with no such feature. The characteristic "chiaroscuro" appearance of the flow fields is a consequence of large velocity magnitude fluctuations - a signature of being close to the self-organization threshold. The parameters are those of Fig. 3.

\section{RESULTS}

By tailoring the generic model Eqs. (1)-(2) to the experimental reality in Sec. III B, we have managed to shrink the parameter space substantially. Nevertheless, the following parameters cannot be predetermined and remain more or less free: the elasticity (diffusivity) $D_{W}$, the noise strength $Q$ (connected with $q$ by Eq. (B5)), the characteristic time $\tau_{W}$ and the coupling $\alpha_{W} \tau_{W} \alpha_{v} \tau_{v}$ controlling the distance from the self-organization threshold Eq. (13). These parameters must be checked for their influence by a rather holistic assessment of the numeric results in the view of experimental data.

Fig. 2 presents snapshots of both fields in the vicinity of the self-organization threshold and for a suitable choice of parameters that conforms to experimental observations. The corresponding channel width dependences of the pumping velocity $v_{x}$ and rms fluctuations of $v_{x}$ (streamwise) and $v_{y}$ (spanwise), all in the middle of the channel, are summarized in Fig. 3 (left). A tiny, completely negligible magnitude of the corotation derivative was assumed at this stage. With the help of Fig. 2 a qualitative comparison can be drawn between the situation in narrow and wider microchannels, which seems to be key for the decrease of pumping coordination in wider channels: the flow vortices have a characteristic size and hardly fit into narrow channels, as opposed to wider channels where the vortices are fully developed. It is fair to say that a disordering effect of noise, in-line with the consideration Eqs. (A6)-(A8) leading to the definition of the persistence length $\lambda_{Q}$, would exist also in the case of a single variable without the continuity requirement, e.g., for a decoupled $\mathbf{W}$ field (even in the coupled case, compare the different morphologies of $\mathbf{v}$ and $\mathbf{W}$ fields in Fig. 2). The conservation of a propagating flux, i.e., the existence of "streams", however, has a strong global influence connecting distant parts of the system, which increases the complexity of the velocity configurations beyond bare diffusion with noise. 

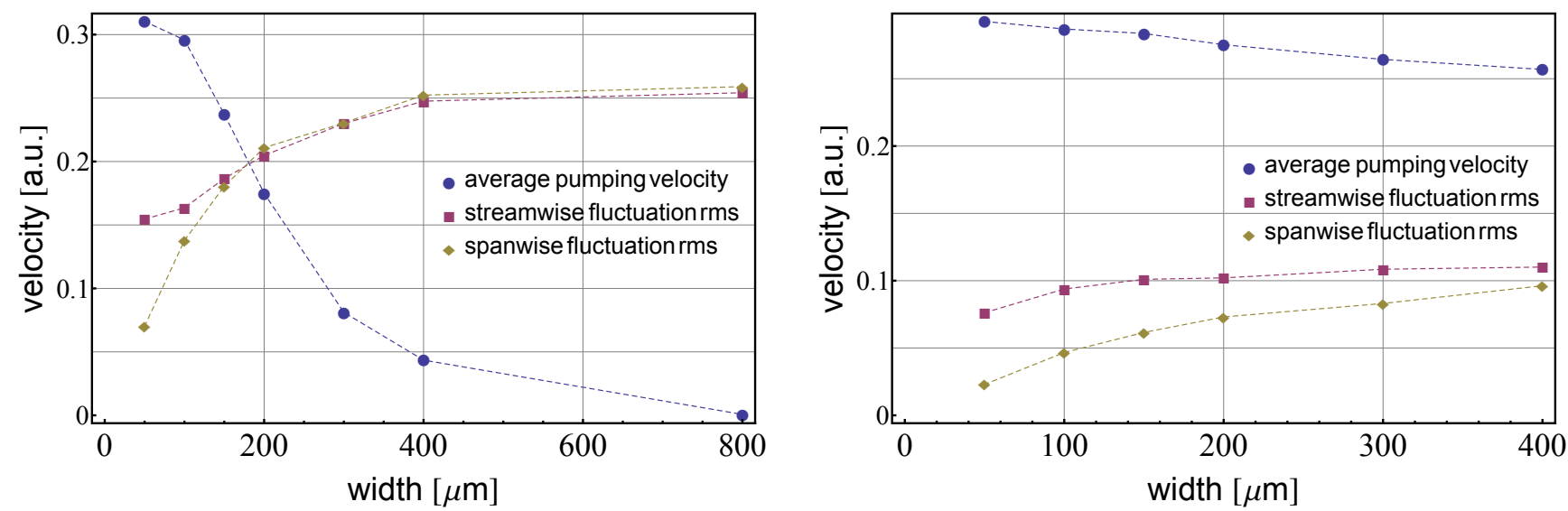

FIG. 3. Near-threshold $\left(\alpha_{W} \tau_{W} \alpha_{v} \tau_{v}=1.2\right)$ channel width dependence of the pumping velocity $\left\langle v_{x}(t)\right\rangle$, as well as streamwise, $\left.\sqrt{\left\langle v_{x}^{2}(t)\right\rangle-\left\langle v_{x}(t)\right\rangle^{2}}\right\rangle$, and spanwise, $\sqrt{\left\langle v_{y}^{2}(t)\right\rangle}$, velocity fluctuations in the middle of the channel $(x=y=0)$. The remaining parameters are $D_{W}=0.005 \mathrm{~mm}^{2} / \tau_{W}, v_{s}=10^{-6} \mathrm{~mm} / \tau_{W}$ (corotation completely negligible), $Q=21 / \tau_{W}$ (left) and $Q=2.1 / \tau_{W}$ (right).
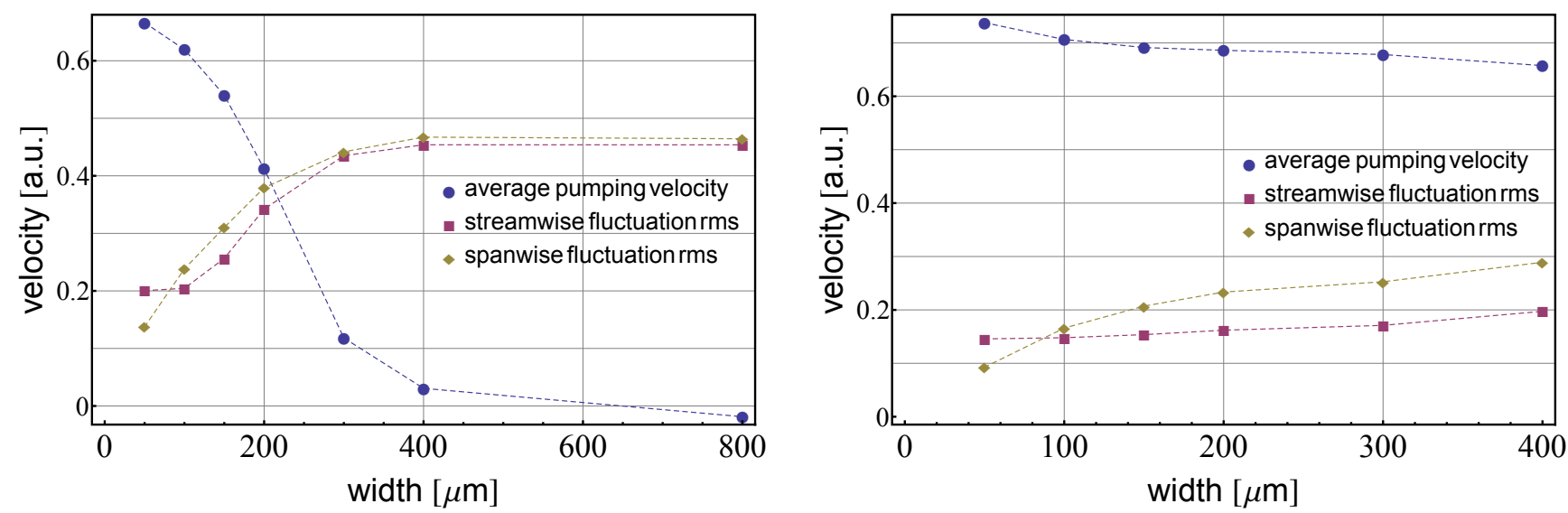

FIG. 4. Far-from-threshold $\left(\alpha_{W} \tau_{W} \alpha_{v} \tau_{v}=33\right)$ channel width dependence of the pumping velocity and streamwise/spanwise velocity fluctuations in the middle of the channel. The remaining parameters are $D_{W}=0.005 \mathrm{~mm}^{2} / \tau_{W}, v_{s}=10^{-6} \mathrm{~mm} / \tau_{W}$ (corotation completely negligible), $Q=210 / \tau_{W}$ (left) and $Q=105 / \tau_{W}$ (right). Note the increased noise strength as compared to Fig. 3.

\section{A. Distance from the self-organization threshold}

Further away from the self-organization threshold, Fig. 4 (left), one can get a width dependence of pumping similar to that near the threshold by suitably increasing the noise strength. A distinction is nevertheless observed: far from the threshold, streamwise fluctuations are significantly weaker, in particular for widths that show efficient pumping. There they are smaller than spanwise fluctuations, which is contrary to the near-threshold case, Fig. 3, and to experimental observations. In Figs. 3 (right) and 4 (right) a smaller noise strength has been chosen to extend the region of efficient pumping and emphasize the difference between stream/spanwise fluctuations of both cases. The observed crossover of fluctuation amplitudes for small channel widths in Fig. 4 (right) is not unexpected - in connection with the characteristic size of vortices, spanwise fluctuations are suppressed in narrow channels.

We can therefore make an important conclusion: the measured dominance of streamwise fluctuations signifies that the system is close to the self-organization threshold. In this case fluctuations of the magnitude are larger, which is revealed also by visually comparing the flow fields in Figs. 5 and 2 (bottom).

Being close to the threshold, the assumption Eq. (A12) is valid, i.e., $\lambda_{Q}$ remains the only relevant characteristic length of the system. In this regime, the crossover from efficient pumping to decay of the self-organization takes place when the channel width $b$ becomes comparable to $\lambda_{Q}$. 


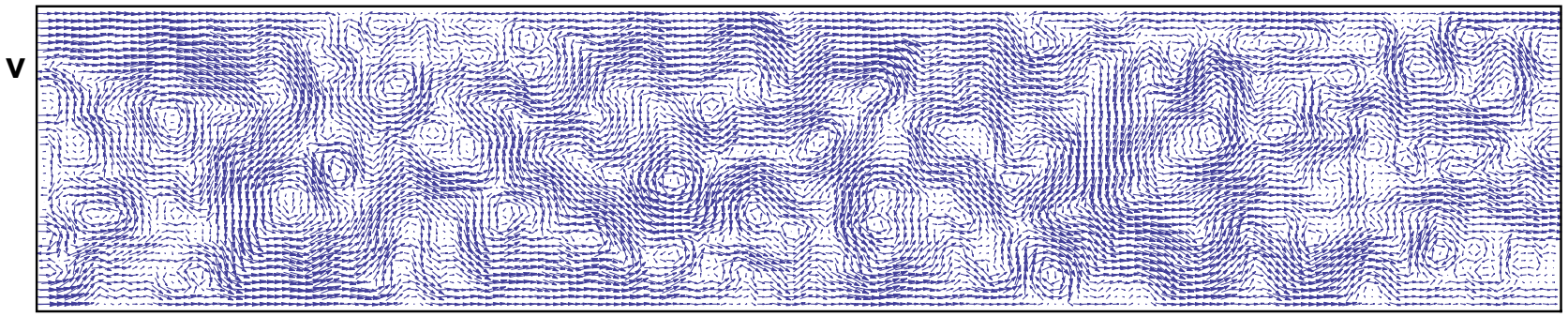

FIG. 5. Snapshots of the fluid velocity field in the microchannel of width $b=400 \mu \mathrm{m}$, far from threshold (parameters as for Fig. 4). Compare the near-threshold velocity field in Fig. 2 (bottom) — in both cases the seed of the random generator as well as the number of how many times it was called are identical, enabling a direct comparison of the flow morphology.

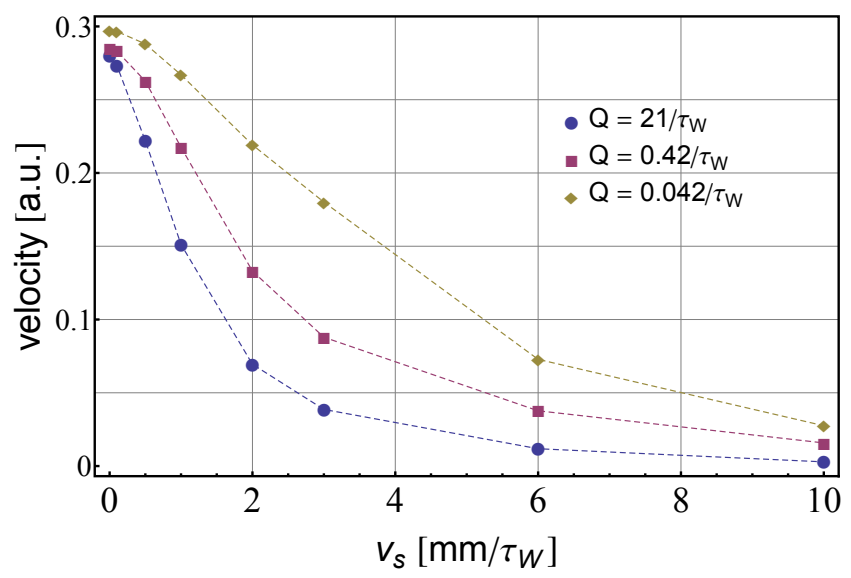

FIG. 6. Dependence of the pumping velocity in the middle of the $200 \mu \mathrm{m}$ channel on the strength of corotation set by the velocity scale $v_{s}$, for noise strength $Q=21 / \tau_{W}$, as well as for $50 \times$ and $500 \times$ weaker noise strengths, showing the destructive effect on the pumping which is not related to noise (remaining parameters as for Fig. 3).

\section{B. Corotation and an upper bound on $\tau_{W}$}

So far the corotation derivative in Eq. (2) has been kept completely negligible by choosing a tiny velocity scale $v_{s}=10^{-6} \mathrm{~mm} / \tau_{W}$, which would correspond to $\tau_{W} \sim 20 \mu \mathrm{s}$. Since a realistic $\tau_{W}$ is estimated to be in the range from $0.1 \mathrm{~s}$ to several $100 \mathrm{~s}$, the corotation is expected to be orders of magnitude stronger in reality. Nevertheless, the results of the model are consistent with the experimental findings already without corotation.

Hence, our strategy is as follows: we keep increasing the parameter $v_{s}$ till we identify a drastic change in the dynamic regime of the model, such that a clear deviation of the modeled pumping velocity from the measured one is observed. This way, we can trace the upper bound of the corotation strength and thus of the characteristic time $\tau_{W}$. A crossover is indeed found around $v_{s} \sim 5 \mathrm{~mm} / \tau_{W}$, as demonstrated by Fig. 6 . Moreover, Fig. 7 illustrates this change in the $200 \mu \mathrm{m}$-wide channel. For the negligible corrotation, Fig. 7 (top), the average pumping velocity in the middle of the channel is $\left\langle v_{x}\right\rangle \approx 0.175$ (dimensionless, arbitrary units) in this case. For $v_{s}=10 \mathrm{~mm} / \tau_{W}$, which corresponds to $\tau_{W} \sim 200 \mathrm{~s}$, the corotation derivative appears to be too strong already, Fig. 7 (bottom), breaking up the flow into smaller vortices and inhibiting pumping, $\left\langle v_{x}\right\rangle \sim 0$. As a contrast, for an order of magnitude weaker corotation, i.e., for $\tau_{W}$ up to $\sim 20 \mathrm{~s}$, the modeled pumping action is only moderately weakened and is still consistent with the experimental data, Fig. 8. It is only that when compared to Fig. 3, the initial decrease of the pumping velocity with channel width appears to be more steady here.

\section{Scaling of noise strength and elasticity}

Here we check explicitly that an efficient pumping takes place also for orders of magnitude lower $D_{W}$, if the noise strength is reduced accordingly, such that the ratio $D_{W} / Q$ of Eq. (A12) is roughly unaffected. Fig. 9 shows pumping for 10-times (left) and 100-times(!) (right) lower elasticity $D_{W}$. Hence, based on the available experimental data it is not possible to extract the absolute value of $D_{W}$, but only its relative magnitude with respect to the noise stength. 

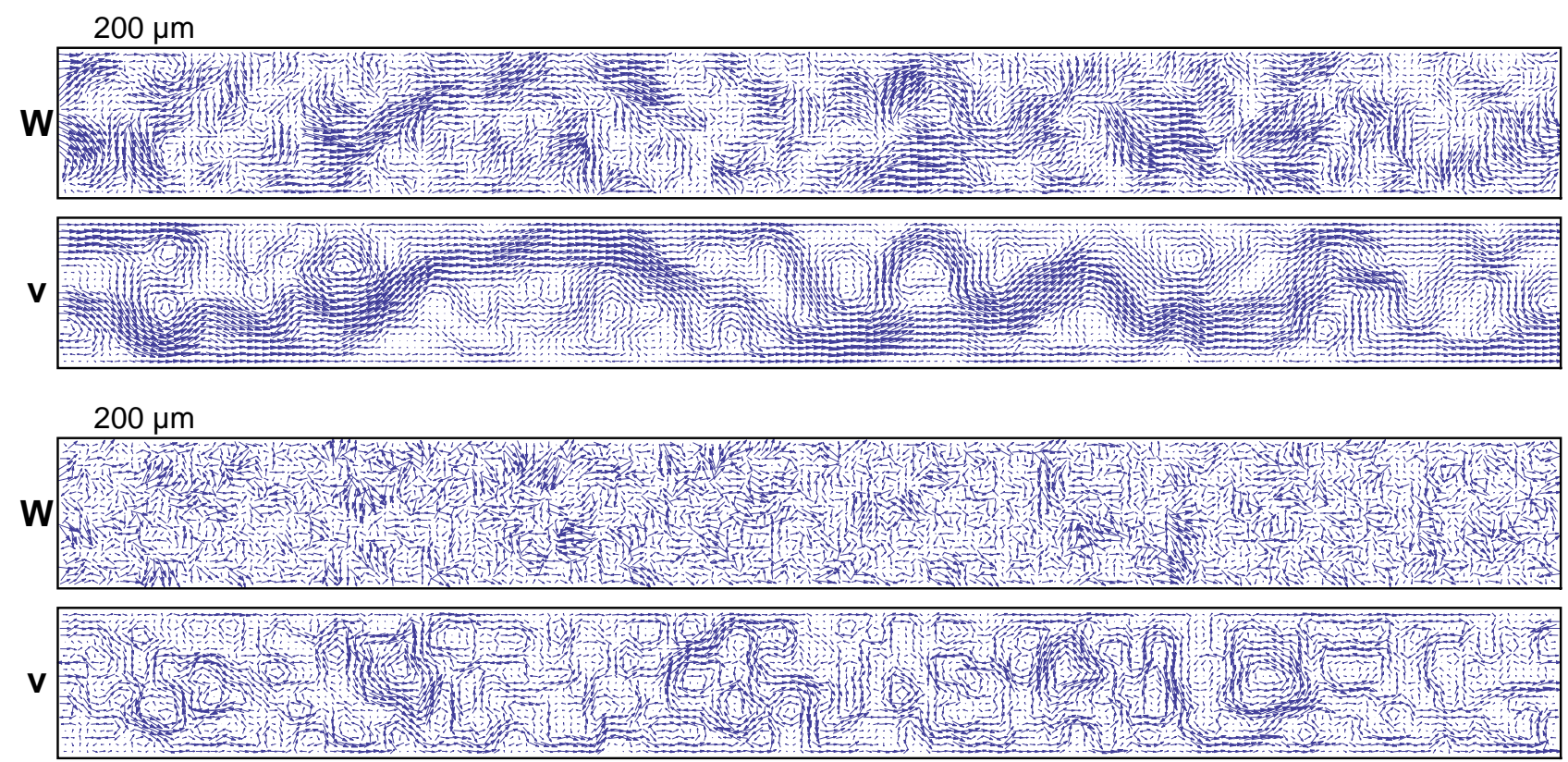

FIG. 7. Snapshots of the $\mathbf{W}$ and $\mathbf{v}$ fields when corotation is negligible (top) (parameters as for Figs. 2 and 3 ) and with $v_{s}=10 \mathrm{~mm} / \tau_{W}$ (bottom), where the corotation is already too strong.
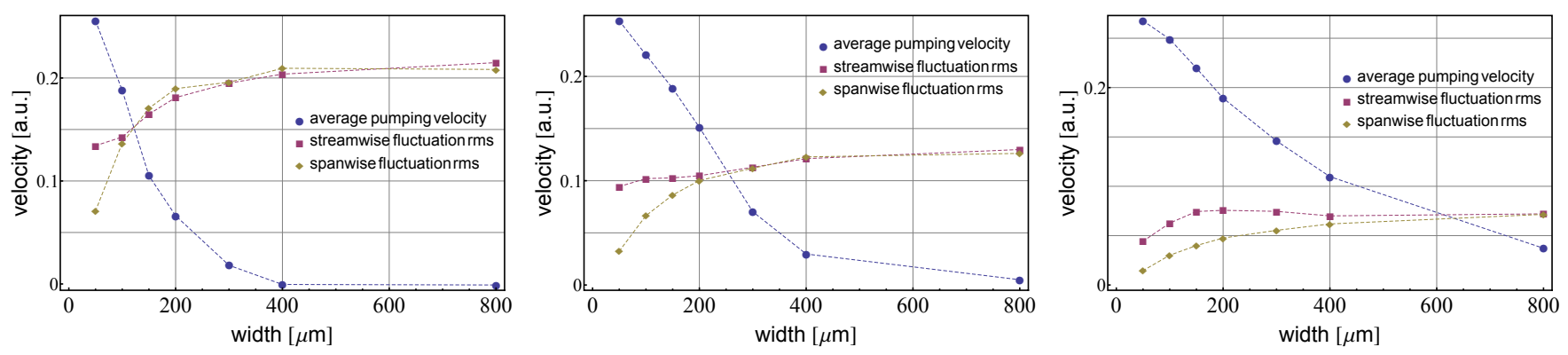

FIG. 8. Channel width dependence of the pumping velocity and streamwise/spanwise velocity fluctuations in the middle of the channel for $v_{s}=1 \mathrm{~mm} / \tau_{W}$ and $Q=21 / \tau_{W}$ (left), $Q=4.2 / \tau_{W}$ (middle) and $Q=0.84 / \tau_{W}$ (right) (remaining parameters as for Fig. 3), showing the influence of corotation that is still compatible with experimental data.

This aspect is closely related to the actual nature of the left-right symmetry breaking discussed in Sec. IIIC. Further experimental input would be needed to tell whether this symmetry breaking is indeed due to the boundary effect as we have described it, or perhaps to a somewhat more delicate bulk effect. In the latter case, the parameter(s) quantifying the nonlinear angle-dependence of the $\mathbf{W}$ bias force would take over the dominant role currently attributed to the elasticity $D_{W}$ of the $\mathbf{W}$ field.

\section{CONCLUSION}

The central result of this study is the introduction of the continuum model Eqs. (1)-(2), capable of describing the bacterial coordination leading to spontaneous pumping. We demonstrated that this self-organization phenomenon can be described by a positive feedback loop between two macroscopic fields - the fluid velocity $\mathbf{v}$ and the average angular velocity $\mathbf{W}$ of the helical bacterial flagella.

Moreover, we showed that the introduced model is also of quantitative relevance. We were able to shrink the number of its parameters and map it to the experimental findings. In particular, we reproduced and explained the experimentally observed reduction of the pumping velocity with increasing width of the microchannel. In the present picture, it is a result of the competition between the ordering effect of the channel boundaries and the destructive influence of noise in the $\mathbf{W}$ variable, which is considered mainly of driven, biologically active origin. Furthermore 

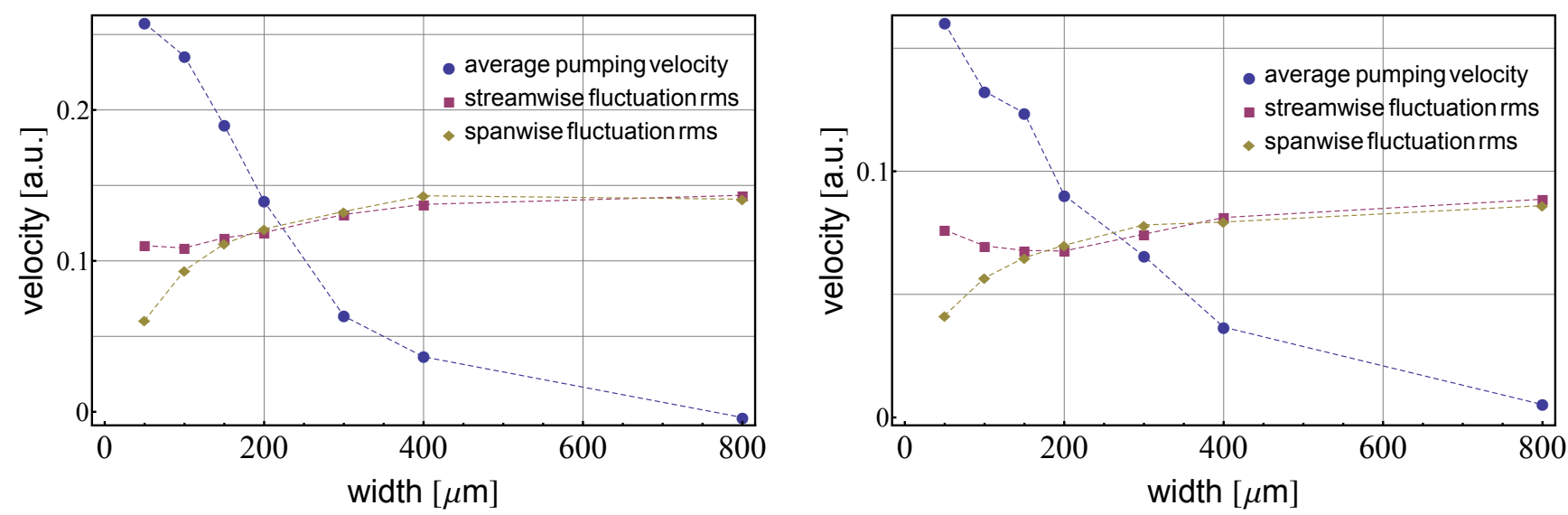

FIG. 9. Channel width dependence of the pumping velocity and streamwise/spanwise velocity fluctuations in the middle of the channel for $D_{W}=5 \times 10^{-4} \mathrm{~mm}^{2} / \tau_{W}$ (left) and $D_{W}=5 \times 10^{-5} \mathrm{~mm}^{2} / \tau_{W}$, while keeping the ratio $D_{W} / Q$ approximately fixed.

we showed that the empirically observed dominance of streamwise velocity fluctuations indicates that the system is close to the threshold of coordination, where magnitude fluctuations of the self-organized order are substantial. This finding is rather unexpected and may hint to a general premise that biological systems are typically rather noisy and are therefore also quite robust against noise.

Several interesting questions have appeared and remain open. We were able to estimate the upper bound of the characteristic dynamic time $\tau_{W}$ of the $\mathbf{W}$ field only very roughly to be of the order $\tau_{W} \sim 100 \mathrm{~s}$, by studying the influence of the corotation derivative of the $\mathbf{W}$ field, i.e., its destructive effect on the pumping. Since corotation was shown not to be crucial to explain the experimental channel-width dependence of the pumping, which can be fully reproduced completely without corotation, the actual $\tau_{W}$ could be much smaller in principle. Nevertheless, the pumping "coordination time" of order of several minutes given in Ref. [5] speaks in favor of the above estimate.

It would be relevant to establish a direct connection between this "coordination time" and the characteristic dynamic time $\tau_{W}$, and ultimately reproduce the empirical channel-width dependence [5] of the former. However, since it appears to be influenced by many factors beyond the present model (including the biological living time of the pumping), a more narrowly targeted time scale, e.g., the initial rise time of the pumping velocity, would be a more relevant measure in the context of the model.

In our view the most important question is the exact nature of the symmetry breaking of the two possible flow directions along the microchannel and the way it is connected to the channel-width dependence of the pumping. It is an empirical fact that this left-right symmetry breaking is caused by the carpet fabrication sequence, although the reasons for this are not clear [5]. If the preferred direction is indeed fixed by the long sides $(y= \pm b / 2)$ of the microchannel as we have assumed, i.e., the average flagella orientation $\mathbf{W}$ is effectively locked there, then the channel-width dependence of the pumping is a rather transparent consequence of the competition between the ordering enforced by the channel boundaries via the $\mathbf{W}$ elasticity, and the disordering by noise. If, on the other hand, the preferred direction were somehow fixed on the entire surface of the bacterial carpet, then a sufficiently nonlinear mechanism of aligning the flagella and locking the left-right symmetry breaking would be required. As discussed in Sec. IIIC, any linear or quasi-linear aligning force would suppress the effect of noise for wider channels, which enhances pumping instead of inhibiting it. In the case of the surface bias, the $y= \pm b / 2$ channel boundaries would be less important and it is less clear how one would explain the experimentally observed width-dependence of the pumping. Spatially correlated (long-wavelength) noise, perhaps resulting from a much stronger elasticity $D_{W}$, could be a relevant feature in this case, and the width-dependence of the pumping could stem from modifications of the spatial spectrum of the fluctuations. In the spirit of Occam's razor, however, the complexity and peculiarity of this scenario speaks against it, and favours the simpler explanation we have taken. Further experimental input would be needed to gain insight into this key physical point.

\section{ACKNOWLEDGMENTS}

The authors thank Martin Horvat for advice re. the pseudo-random number generator. D.S. acknowledges the support of the Slovenian Research Agency, Grants J1-7435 and J1-7441. 


\section{Appendix A: Reduced solutions and characteristic lengths}

The mapping of the model to the realistic situation showed that the diffusivity $D_{W}$ of the $\mathbf{W}$ field and the noise strength $q$ are physically the most important parameters. In the regime of weak supercritical coupling their role can be explored more closely. Taking into account the quasi-stationarity of $\mathbf{v}$, neglecting the small in-plane viscosity $D_{v}$, considering the weak coupling conditions Eq. (7) (due to the choice Eq. (14) they also imply $W_{0}^{2} \ll 1, v_{0}^{2} \ll 1$ ), omitting noise and assuming a fixed direction $\mathbf{v} \| \mathbf{W}$, the linearized Eqs. (1) and (2) for $v=v_{0}+\delta v, W=W_{0}+\delta W$ in the lowest nontrivial order of $v_{0}, W_{0}$ read

$$
\begin{gathered}
0=\tau_{W} D_{W} \nabla^{2} \delta v+\left[\left(\alpha_{W} \tau_{W} \alpha_{v} \tau_{v}-1\right)-3 v_{0}^{2}\left(\tau_{v} \gamma_{v}+\frac{\tau_{W} \gamma_{W}}{\tau_{v}^{2} \alpha_{v}^{2}}\right)\right] \delta v, \\
\tau_{W} \frac{\partial \delta W}{\partial t}=\tau_{W} D_{W} \nabla^{2} \delta W+\left[\left(\alpha_{W} \tau_{W} \alpha_{v} \tau_{v}-1\right)-3 W_{0}^{2}\left(\tau_{W} \gamma_{W}+\frac{\tau_{v} \gamma_{v}}{\tau_{W}^{2} \alpha_{W}^{2}}\right)\right] \delta W .
\end{gathered}
$$

For $v_{0}=W_{0}=0$, Eq. (A2) reproduces the linear rate Eq. (12) of the self-organization growth or decay, depending on whether we are above or below the threshold Eq. (13). In the case of the ordered ground state, $W_{0}$ and $v_{0}$ are given by Eqs. (8)-(9) and from Eqs. (A1) and (A2) we then get

$$
\begin{aligned}
0 & =\tau_{W} D_{W} \nabla^{2} \delta v-2\left(\alpha_{W} \tau_{W} \alpha_{v} \tau_{v}-1\right) \delta v, \\
\tau_{W} \frac{\partial \delta W}{\partial t} & =\tau_{W} D_{W} \nabla^{2} \delta W-2\left(\alpha_{W} \tau_{W} \alpha_{v} \tau_{v}-1\right) \delta W .
\end{aligned}
$$

Eqs. (A3) and (A4) define a correlation length $\xi$,

$$
\xi^{2}=\frac{\tau_{0} D_{W}}{2} .
$$

In bulk and in the absence of noise, $\xi$ would be the characteristic size of vortices in the $\mathbf{v}$ and $\mathbf{W}$ fields. We see that it depends on the ratio of the elasticity (diffusivity) $D_{W}$ and the linear growth rate $1 / \tau_{0}$, describing the distance from the threshold, Eq. (12).

However, another characteristic length, related to noise - a "persistence" length $\lambda_{Q}$ of the self-organized order can be defined by

$$
\lambda_{Q}^{2}=2 \pi^{2} \frac{D_{W}}{Q-4 / \tau_{0}} .
$$

In deriving Eq. (A6) we have added a scalar noise term to Eq. (A4) and considered a single one-dimensional $k_{0}=\pi / b$ Fourier mode $\delta W(x, t)=\delta W_{0}(t) \sin k_{0} x$ in the range $x \in[0, b]$ corresponding to the width of the channel,

$$
\delta \dot{W}_{0}+\left(D_{W} k_{0}^{2}+\frac{2}{\tau_{0}}\right) \delta W_{0}=\zeta_{0}(t)
$$

where $\zeta_{0}(t)=(2 / b) \int_{0}^{b} d x \sin k_{0} x \zeta(x, t)$ and $\left\langle\zeta_{0}(t) \zeta\left(t^{\prime}\right)\right\rangle=Q \delta\left(t-t^{\prime}\right)$. The solution is

$$
\left\langle\delta W_{0}^{2}(t)\right\rangle=\frac{Q}{2\left(D_{W} k_{0}^{2}+2 / \tau_{0}\right)}
$$

and at a given noise strength $Q$ depends on the channel width $b$. Generally, the ordering of the flagella will be destroyed by noise when $\left\langle\delta W_{0}^{2}(t)\right\rangle$ is of order unity. Requiring $\left\langle\delta W_{0}^{2}(t)\right\rangle=1$ in Eq. (A8), expressing from it the channel width and calling it $\lambda_{Q}$, Eq. (A6) follows. The ordering of the flagella thus persists over the length scale given by $\lambda_{Q}$. This length can be interpreted also as the scale of smallest vortices generated by the noise of strength $Q$. For the pumping self-organization to decay with increasing width of the microchannel in this reduced one-dimensional model, $Q>4 / \tau_{0}$ must hold. In Sec. IV C we have shown, however, that in the full model we get a similar width dependence of the pumping also for noise strengths that are significantly lower than this margin, which speaks for the importance of transverse fluctuations.

Transverse fluctuations (spanwise fluctuations, fluctuations of $W_{y}$ and $v_{y}$ ) cannot be estimated as easily. They involve the incompressibility condition and are inherently two-dimensional. Through this coupling, they also acquire the "gap-like" ("massive") character, involving variations of the magnitudes again. In a terminally simplified picture, where we neglect all of this and treat $\mathbf{W}$ as the sole variable, we can write down a simple equation, analogous to Eq. (A7), for the lowest Fourier mode of the angle of $\mathbf{W}$ with respect to the $x$ axis, $\varphi(x, t)=\varphi_{0}(t) \sin k_{0} x$,

$$
\dot{\varphi}_{0}+D_{W} k_{0}^{2} \varphi_{0}=\zeta_{0}(t)
$$


with the solution

$$
\left\langle\varphi_{0}^{2}(t)\right\rangle=\frac{Q}{2 D_{W} k_{0}^{2}} .
$$

Requiring $\left\langle\varphi_{0}^{2}(t)\right\rangle=1$ again, a "persistence" length $\lambda_{Q}^{\perp}$ for transverse fluctuations is defined this time,

$$
\left(\lambda_{Q}^{\perp}\right)^{2} \sim 2 \pi^{2} \frac{D_{W}}{Q} .
$$

Due to the neglected magnitude variations, this length is somewhat larger in principle. In the experimentally relevant regime, however, $\lambda_{Q}^{\perp}$ is much shorter than $\xi$ as will turn out in Sec. IV A, so that the magnitude variations are not particularly costly and the estimate Eq. (A11) is good, while moreover

$$
\lambda_{Q}^{2}=\left(\frac{1}{\left(\lambda_{Q}^{\perp}\right)^{2}}-\frac{1}{\pi^{2} \xi^{2}}\right)^{-1} \approx\left(\lambda_{Q}^{\perp}\right)^{2} \approx 2 \pi^{2} \frac{D_{W}}{Q} .
$$

In this most relevant regime, the flow morphology is governed by the competition between diffusion (elasticity) of the $\mathbf{W}$ field and noise acting on it.

\section{Appendix B: Method}

The dynamic equations Eqs. (1) and (2) are advanced in time by the Euler method on a 2D equilateral (square) mesh discretizing the channel in $x$ and $y$ directions. As explained in Sec. III B, the velocity field is quasi-stationary. We therefore iterate it to adequate convergence at every time step of the $\mathbf{W}$ equation. The incompressibility condition $\nabla \cdot \mathbf{v}=0$ is satisfied by an artificial compressibility approach [44], i.e., using a dynamic equation for the density of the fluid,

$$
\frac{\partial \rho}{\partial t}+\nabla \cdot \rho \mathbf{v}=0, \quad \rho(\mathbf{r})=K p(\mathbf{r})
$$

where $K$ is the "compressibility" parameter. Eq. (B1) is iterated simultaneously with the velocity equation Eq. (1) on a staggered grid [44] to avoid spatial oscillations. When performing the discretization, care was taken to identically conserve the volume fluxes between computational cells.

The parameter $K$ must be sufficiently large, such that the incompressibility condition is enforced to an adequate accuracy $\left(|\nabla \cdot \mathbf{v}| /(v / b)<10^{-4}\right.$ proved to be sufficient). A dilatation viscosity term $\zeta \nabla \nabla \cdot \mathbf{v}$ is added to the right-handside of Eq. (1) to damp the artificial density waves on the way to reaching the quasi-stationary $\mathbf{v}(x, y)$ and $p(x, y)$ solutions. A near-optimum value of $\zeta$ is chosen, such that the density field is optimally equilibrated in the available number of iterations. The adequacy of the number of iterations and the magnitude of $K$ is verified empirically by e.g. doubling the values and making sure that this has no significant effect on the observables.

The stochastic force $\boldsymbol{\zeta}(x, y, t)$ in Eq. (2) is a pseudo-random number (generated by the Mersenne Twister pseudorandom generator) satisfying

$$
\left\langle\left|\boldsymbol{\zeta}(x, y, t) \cdot \boldsymbol{\zeta}\left(x^{\prime}, y^{\prime}, t^{\prime}\right)\right|\right\rangle=(2 q) \Pi_{\Delta x}\left(x-x^{\prime}\right) \Pi_{\Delta y}\left(y-y^{\prime}\right) \chi_{\Delta t}\left(t-t^{\prime}\right),
$$

where $\Pi_{\Delta x}, \Pi_{\Delta y}$ are zero-centered boxcar functions of widths equal to the grid spacings $\Delta x=\Delta y$, and $\chi_{\Delta t}(\tau)$ is a dimensionless time autocorrelation function satisfying $\chi_{\Delta t}(0)=1$. The factor of 2 in $2 q$ stands for the two components of $\boldsymbol{\zeta}$, such that $q$ represents the scalar noise strength. To keep the spatial characteristic (autocorrelation) of the noise exactly the same for all microchannel widths, both grid spacings were fixed in all cases, i.e., the number of $y$ mesh points was proportional to the channel width.

To avoid possible influence of temporal periodicity, the noise was not applied in regular time intervals. Instead, in every mesh point a new noise value would be assigned with probability $d t / \Delta t$, where $\Delta t \geq d t$ is the average noise application time interval and $d t$ is the computation time step. Unless $d t=\Delta t$, the resulting time autocorrelation $\chi_{\Delta t}(\tau)$ is not simple. In the limit of continuous time, i.e., when $d t / \Delta t \equiv p_{t} d t \ll 1$, one gets $\chi_{\Delta t}(\tau)=\exp \left(-p_{t} \tau\right)-$ $p_{t} \tau \Gamma\left(0, p_{t} \tau\right)$, where $\Gamma$ is the incomplete gamma function. Using the Wiener-Khinchin theorem, in every mesh point the noise power $(P)$ per unit frequency $(\omega)$ is then $\Phi(\omega) \equiv d P / d \omega=(2 q / \pi) \int_{0}^{\infty} d \tau \cos \omega \tau \chi_{\Delta t}(\tau)$, with the result

$$
\Phi(\omega)=\frac{2 q}{2 \pi p_{t}}\left(\frac{p_{t}}{\omega}\right)^{2} \ln \left[1+\left(\frac{\omega}{p_{t}}\right)^{2}\right]
$$


and its low-frequency limit

$$
\Phi(0)=\frac{2 q}{2 \pi p_{t}}=\frac{2 Q}{\pi} .
$$

Lastly, we have written down the explicit connection

$$
Q=q /\left(2 p_{t}\right)
$$

to the commonly assumed case of perfectly uncorrelated noise with strength $Q$, which was also used in the definitions Eqs. (A6) and (A11).

Keeping the physical parameter $p_{t}=1 / \Delta t$ fixed guarantees that the noise time autocorrelation (power spectrum) is strictly independent of possible time step changes, provided that $d t / \Delta t \ll 1$ holds for all time steps $d t$. In practice this is not necessarily satisfied, but since in Eq. (2) the noise is integrated over time, its low frequency components contribute the most, and it is these components that are less affected by the coarseness of $d t / \Delta t$.

[1] N. Darnton, L. Turner, K. Breuer, H. C. Berg, Biophys. J., 2004, 86, 1863.

[2] M. J. Kim, T. R. Powers, Phys. Rev. E, 2004 69, 061910.

[3] M. J. Kim, K. S. Breuer, J. Fluids Eng., 2007, 129, 319.

[4] E. B. Steager, C.-B. Kim, M. J. Kim, Phys. Fluids, 2008, 20, 073601.

[5] M. J. Kim, K. S. Breuer, Small, 2008, 4, 111.

[6] Y. Wu, B. G. Hosu, H. C. Berg, Proc. Natl. Acad. Sci. U.S.A., 2011, 108, 4147.

[7] Y.-T. Hsiao, J.-H. Wang, K.-T. Wu, J. Tsai, C.-H. Chang, W.-Y. Woon, Appl. Phys. Lett., 2014, $105,203702$.

[8] H. Kim, U. K. Cheang, D. Kim, J. Ali, M. J. Kim, Biomicrofluidics, 2015, 9, 024121.

[9] Z. Gao, H. Li, X. Chen, H. P. Zhang, Lab Chip, 2015, 15, 4555.

[10] Y.-T. Hsiao, K.-T. Wu, N. Uchida, W.-Y. Woon, Appl. Phys. Lett., 2016, 108, 183701.

[11] F. G. Woodhouse, A. Forrow, J. B. Fawcett, J. Dunkel, Proc. Natl. Acad. Sci. U.S.A., 2016, $19,8200$.

[12] F. G. Woodhouse, J. Dunkel, Nat. Commun., 2017, 8, 15169.

[13] F. Nédélec, T. Surrey, A. C. Maggs, S. Leibler, Nature, 1997, 389, 305.

[14] K. Kinosita Jr, R. Yasuda, H. Noji, K. Adachi, Phil. Trans. R. Soc. Lond. B, 2000, 355, 473.

[15] T. Surrey, F. Nédélec, S. Leibler, E. Karsenti, Science, 2001, 292, 1167.

[16] V. Schaller, C. Weber, C. Semmrich, E. Frey, A. R. Bausch, Nature, 2010, 467, 73.

[17] V. Schaller, C. A. Weber, B. Hammerich, E. Frey, A. R. Bausch, Proc. Natl. Acad. Sci. U.S.A., $2011,108,19183$.

[18] D. A. Head, W. Briels, G. Gompper, BMC Biophysics, 2011, 4, 18.

[19] Y. Sumino, K. H. Nagai, Y. Shitaka, D. Tanaka, K. Yoshikawa, H. Chaté, K. Oiwa, Nature, $2012,483,448$.

[20] Q. Li, G. Fuks, E. Moulin, M. Maaloum, M. Rawiso, I. Kulic, J. T. Foy, N. Giuseppone, Nat. Nanotech., $2015,10,161$.

[21] K. Watanabe, J. Wakita, H. Itoh, H. Shimada, S. Kurosu, T. Ikeda, Y. Yamazaki, T. Matsuyama, M. Matsushita, J. Phys. Soc. Jpn., 2002, 71, 650 .

[22] L. H. Cisneros, R. Cortez, C. Dombrowski, R. E. Goldstein, J. O. Kessler, Exp. Fluids, 2007, 43, 737.

[23] H. P. Zhang, A. Be'er, E.-L. Florin, H. L. Swinney, Proc. Natl. Acad. Sci. U.S.A., 2010, 107, 13626.

[24] D. Svenšek, H. Pleiner, H. R. Brand, Phys. Rev. Lett., 2013, 111, 228101.

[25] S. Zhou, A. Sokolov, O. D. Lavrentovich, I. S. Aranson, Proc. Natl. Acad. Sci. U.S.A., 2014, $111,1265$.

[26] I. H. Riedel, K. Kruse, J. Howard, Science, 2005, 309, 300.

[27] J. Elgeti, U. B. Kaupp, G. Gompper, Biophys. J., 2010, 99, 1018.

[28] J. Buhl, D. J. T. Sumpter, I. D. Couzin, J. J. Hale, U. Despland, E. R. Miller, S. J. Simpson, Science, $2006,312,1402$.

[29] C. M. Topaz, M. R. D'Orsogna, L. Edelstein-Keshet, A. J. Bernoff, PLoS Comput. Biol., 2012 , 8, e1002642.

[30] S. V. Viscido, J. K. Parrish, D. Grünbaum, Mar. Ecol. Prog. Ser., 2004, 273, 239.

[31] Y. Katz, K. Tunstrom, C. C. Ioannou, C. Huepe, I. Couzin, Proc. Natl. Acad. Sci. U.S.A., 2011, $108,18720$.

[32] M. Ballerini, N. Cabibbo, R. Candelier, A. Cavagna, E. Cisbani, I. Giardina, V. Lecomte, A. Orlandi, G. Parisi, A. Procaccini, M. Viale, V. Zdravkovic, Proc. Natl. Acad. Sci. U.S.A., 2008, 105, 1232.

[33] A. Cavagna, A. Cimarelli, I. Giardina, G. Parisi, R. Santagati, F. Stefanini, M. Viale, Proc. Natl. Acad. Sci. U.S.A., 2010, 107, 11865.

[34] R. Lukeman, Y. Li, L. Edelstein-Keshet, Proc. Natl. Acad. Sci. U.S.A., 2010, 107, 12576.

[35] D. J. G. Pearce, A. M. Miller, G. Rowlands, and M. S. Turner, Proc. Natl. Acad. Sci. U.S.A., $2014,111,10422$.

[36] F. Ginelli, F. Peruani, M.-H. Pillot, H. Chaté, G. Theraulaz, R. Bon, Proc. Natl. Acad. Sci. U.S.A., $2015,112,12729$.

[37] D. Marenduzzo, E. Orlandini, M. E. Cates, J. M. Yeomans, Phys. Rev. E, 2007, 76, 031921.

[38] K. Kruse, J. F. Joanny, F. Jülicher, J. Prost, K. Sekimoto, Eur. Phys. J. E, 2005, 16, 5.

[39] H. R. Brand, H. Pleiner, D. Svenšek, Eur. Phys. J. E, 2011, 34, 128.

[40] H. Pleiner, D. Svenšek, H. R. Brand, Eur. Phys. J. E, 2013, 36, 135.

[41] T. Vicsek, A. Czirók, E. Ben-Jacob, I. Cohen, O. Shochet, Phys. Rev. Lett., 1995, 75, 1226. 
[42] J. Toner and Y. Tu, Phys. Rev. Lett., 1995, 75, 4326.

[43] P. G. De Gennes, J. Prost, The Physics of Liquid Crystals, Clarendon Press, Oxford, 1995.

[44] C. A. J. Fletcher, Computational Techniques for Fluid Dynamics, Volume II, 2nd Edition, Springer-Verlag, New York, Berlin, Heidelberg, 1991. 